\title{
Frontostriatal Involvement in Task Switching Depends on Genetic Differences in D2 Receptor Density
}

\author{
Christine Stelzel, ${ }^{1,2,3}$ Ulrike Basten, ${ }^{1,2}$ Christian Montag, ${ }^{4}$ Martin Reuter, ${ }^{4}$ and Christian J. Fiebach ${ }^{1,2,5}$ \\ ${ }^{1}$ Department of Psychology, University of Heidelberg, D-69117 Heidelberg, Germany, ${ }^{2}$ Department of Neuroradiology, University of Heidelberg, D-69120 \\ Heidelberg, Germany, ${ }^{3}$ Department of Psychiatry and Psychotherapy, Charité Universitätsmedizin, D-10117 Berlin, Germany, ${ }^{4}$ Department of Psychology, \\ University of Bonn, D-53111 Bonn, Germany, and 5 Department of Psychology, Goethe University Frankfurt, D-60054 Frankfurt am Main, Germany
}

Recent studies suggest an association of dopamine D2 receptor (DRD2) availability with flexibility in reward-based learning. We extend these results by demonstrating an association of genetically based differences in DRD2 density with the ability to intentionally switch between nonrewarded tasks: noncarriers of the A1 allele of the DRD2/ANKK1-TaqIa polymorphism, associated with higher DRD2 density, show increased task-switching costs, increased prefrontal switching activity in the inferior frontal junction area, and increased functional connectivity in dorsal frontostriatal circuits, relative to A1 allele carriers. A DRD2 haplotype analysis in the same sample confirmed these results, indicating an association between high D2 density and increased task-switching effort. Our results provide evidence that converges with that from association studies relating increased D2 density to deficits in cognitive flexibility in schizophrenia. We suggest that individual differences in striatal D2 signaling in healthy humans modulate goal-directed gating to prefrontal cortex, thus leading to individual differences in switching intentionally to newly relevant behaviors.

\section{Introduction}

Flexible adaptation of behavior to changing environments is essential for success in today's multitasking society and has been related to the prefrontal cortex (Fuster, 2001; Miller and Cohen, 2001). While clinical disorders such as schizophrenia are associated with severe deficits in cognitive flexibility (Goldberg and Weinberger, 1988), substantial variation is also found in healthy humans (Miyake et al., 2000). An important modulator of behavioral flexibility is the individual availability of prefrontal dopamine (Cools and Robbins, 2004). The dopamine D2 receptor (DRD2), in particular, was associated with flexible, rewarddependent behavioral adaptation in pharmacological studies in rodents (Floresco et al., 2006) and in human imaging genetics studies (Klein et al., 2007; Jocham et al., 2009).

Humans, however, are also capable of flexibly applying different task rules without long-term reward-based learning. Cognitive flexibility based exclusively on external instructions or internal intentions is a highly heritable ability that differentiates humans from many other species (Friedman et al., 2008). Experimentally, this ability is assessed in task-switching paradigms (Jersild, 1927), where the need to switch between two tasks is indicated by task cues associated with the respective tasks. Individual flexibility is measured as the perfor-

Received March 2, 2010; revised Aug. 20, 2010; accepted Aug. 24, 2010.

This work was funded by an Emmy-Noether grant from the German Research Foundation (DFG FI 848/3-1) and by the German Excellence Initiative. We thank Martin Bendszus for his support of this research.

The authors declare no conflict of interest.

Correspondence should be addressed to Christine Stelzel, Department of Psychiatry and Psychotherapy, Division of Mind and Brain Research, Charité Universitätsmedizin Berlin, Charité Campus Mitte, Charitéplatz 1, D-10117 Berlin. E-mail: christine.stelzel@charite.de.

DOI:10.1523/JNEUROSCI.1062-10.2010

Copyright $\odot 2010$ the authors $\quad 0270-6474 / 10 / 3014205-08 \$ 15.00 / 0$ mance decrement in switching relative to task repetitions (Monsell, 2003).

While reward-based flexibility recruits orbitofrontal and ventral striatal brain regions (Wallis, 2007), rule-based task switching is associated with more posterior prefrontal (Brass and von Cramon, 2002) and dorsal striatal (Gu et al., 2008) regions. Initial evidence from pharmacological (Mehta et al., 2004) and patient (Cools et al., 2001; Kehagia et al., 2009) work suggests an association of dopamine and rule-based switching, but it is presently unclear whether genetically determined differences in DRD2mediated dopamine signaling affect the neural implementation of rule-based task switching analogous to reward-dependent behavioral adaptations. We thus assessed the impact of D2 receptor density on task-switching performance and underlying frontostriatal neural circuits (Frank et al., 2001) using the DRD2/ ANKK1-TaqIa polymorphism (rs1800497). DRD2/ANKK1-TaqIa modulates DRD2 density such that carriers of the A1 allele (A1+) have a 30\% reduced DRD2 density compared to homozygous A2 allele carriers (Ritchie and Noble, 2003). This effect is particularly prominent in the striatum, but also affects prefrontal cortex (Noble et al., 1997).

Several disorders have been associated with changes in the DRD2 system (Noble, 2003; Bowirrat and Oscar-Berman, 2005). Most prominently, increased striatal DRD2 density is characteristic for schizophrenia (Wong et al., 1986; Abi-Dargham et al., 2000), which is associated with impaired cognitive flexibility (Rodríguez-Sánchez et al., 2005; Thoma et al., 2007). Increased DRD2 availability is further associated with changes in prefrontal dopamine levels and cognitive inflexibility in transgenic mice (Kellendonk et al., 2006), suggesting that striatal DRD2 density might also modulate switching performance in human prefrontal cortex. To test this hypothesis, we examined how long-term ge- 
netic differences in the DRD2 system relate to individual differences in rule-based flexibility in terms of the invested neural effort. We additionally explored the effects of a recently reported DRD2 haplotype block (Markett et al., 2010) to further generalize our conclusions with respect to the role of DRD2 in cognitive flexibility.

\section{Materials and Methods}

Participants. All 48 participants were native Germans of Caucasian ethnicity, without neurological or psychiatric history, and had normal or corrected-to-normal vision (24 females, age: $M=22.0, \mathrm{SD}=1.99 ; 24$ males, age: $M=$ 22.6, SD $=1.99$ ). Participants were selected from a larger sample of genotyped individuals (Stelzel et al., 2009), to include $24 \mathrm{~A} 1+$ and 24 A1 - individuals with equal gender distribution. As the present experiment was part of a larger research project, the task-switching paradigm was administered in one fMRI session together with a dual-task paradigm (see below) and one other experiment that followed the task-switching experiment in all subjects and thus did not have any effect on the present experiment. The study was approved by the local ethics committee of the Medical School of the University of Heidelberg, and participants gave written consent according to the Declaration of Helsinki.

Behavioral procedure. Participants performed two different tasks on visually presented number stimuli depending on a task cue presented $300 \mathrm{~ms}$ before the number stimuli. The task cue remained on the screen until the end of the trial (Fig. 1a), and participants were to respond fast and accurately. A diamond cue indicated that participants were to decide whether the number stimulus was smaller or larger than five. A square indicated to decide whether the number was odd or even. The number stimulus was presented in the center of the cue symbol for $1700 \mathrm{~ms}$, followed by a variable inter trial interval of 2, 4, or $6 \mathrm{~s}$. In addition to the sequence of the two tasks, the location of the task cues was varied. Cues were presented either to the left or the right of the fixation cross, indicating the response hand for the present trial. If cue and stimulus appeared to the left of the fixation cross, the response was to be given with the middle or index finger of the left hand, if it appeared to the right of the fixation cross, participants were to respond with the right middle or index finger. Participants responded with the left finger of the respective response hand if the stimulus was smaller than five or even and with the right finger if it was greater than five or odd. The task sequence was pseudorandomized with the restriction that not more than three task repetitions, two stimulus repetitions, and three response repetitions occurred in sequence. Transitions between conditions were balanced evenly. Participants received two blocks of training with the tasks directly before the MRI session. These training blocks were included in the analyses of the behavioral DRD2 $\times$ task switching interaction to increase power. Note that we did not obtain results from the training for 7 participants, so analyses including behavioral training data were done for 41 participants only. During fMRI measurement, the two task-switching runs were separated by two dualtask runs, in which the two tasks of the task-switching experiment were presented temporally overlapping and subjects had to respond to both stimuli within one trial. As the order of the different runs was constant across subjects, no differential carry-over effects of the dual-task performance on the second task-switching run is expected.

Genetic analyses. DNA was extracted from buccal cells. Automated purification of genomic DNA was conducted by means of the MagNA Pure LC system using a commercial extraction kit (MagNA Pure LC DNA isolation kit; Roche Diagnostics). Genotyping was performed by real-time PCR using fluorescence melting curve detection analysis by means of the Light Cycler System (Roche Diagnostics). Details of the PCR protocols, primers, and hybridization probes were described previously (Markett et al., 2010). Carriers of the DRD2/ANKK1-TaqIa A1 allele (A1A1, A1A2) were assigned to the $\mathrm{A} 1+$ group, and noncarriers of the $\mathrm{A} 1$ allele (A2A2) were assigned to the A1 - group. Note that the A1A1 subtype is extremely rare-about $3 \%$ in healthy Caucasians (Noble, 2000a) — and therefore is commonly grouped with the A1A2 subtype in genetic association studies. In Caucasian samples, also the combined $\mathrm{A} 1+$ subtype is less frequent than the A1- subtype, with about $28 \%$ carrying the A1 allele (Noble, 2000b). The DRD2/ANKK1-TaqIa SNP (rs1800497) is located $<10 \mathrm{~kb}$ downstream of the DRD2 gene and actually lies in the protein-coding region of the adjacent ANKK1 gene. However, it has been shown that due to a linkage disequilibrium, the DRD2/ ANKK1-TaqIa SNP can be considered a reliable marker for variations in D2 receptor density (Zhang et al., 2007).

Haplotype analysis. Haplotype blocks (Markett et al., 2010) included the DRD2/ANKK1-TaqIa polymorphism (rs1800497), as well as two other DRD2 gene polymorphisms, i.e., DRD2 c957t (rs6277) and rs2283265, that have been related to decreased D2 receptor density and an altered DRD2 subtype proportion (i.e., relatively more receptors postsynaptically than presynaptically), respectively. Linkage analyses between SNPs and construction of haplotype blocks were conducted by means of Haploview 3.32 (http://www.broad.mit.edu/mpg/haploview/ index.php). Individual haplotypes were calculated with PHASE, version 2.1. PHASE implements a Bayesian statistical method for reconstructing haplotypes from population genotype data (Stephens et al., 2001). The construction of haplotype blocks was conducted via the solid spine of LD method. Note that the composite haplotype measure is statistically not equivalent with a situation in which each SNP is included in the analysis as an independent factor, i.e., effects do not sum up in a linear fashion as dependencies across different SNPs are taken into account (MeyerLindenberg and Weinberger, 2006). Hence, although not completely independent of the DRD2/ANKK1-TaqIa factor, the categorization of subjects according to the haplotype provides a relevant additional 
between-subject factor to test for the generality of the findings from the single SNP analysis.

fMRI procedure. All images were acquired with a 3 tesla Siemens Trio MRI scanner equipped with a fast gradient system for echo-planar imaging. We used a birdcage head coil and stabilized participants with cushions to restrict head motion comfortably. Functional images were acquired in two runs, using a whole-brain one-shot gradient-echo, echoplanar sequence $(\mathrm{TE}=30 \mathrm{~ms}$, matrix size $=64 \times 64, \mathrm{FOV}=24 \mathrm{~cm}$, flip angle $=83^{\circ}$, TR $=2 \mathrm{~s}$ ). Each functional volume consisted of 32 axial slices with $3 \mathrm{~mm}$ thickness and $1 \mathrm{~mm}$ interslice gap. We also acquired a structural T1-weighted 3-D MPRAGE scan at the end of the experiment (matrix size $256 \times 256$, slice thickness: $1.0 \mathrm{~mm}$, flip angle: $30^{\circ}$ ). In addition, we acquired a 2-D T1-weighted image using the same slice prescription as for functional scans. Anatomical images were used for the normalization of the functional data to the Montreal Neurological Institute (MNI) atlas space.

fMRI data analyses. All analyses were performed with SPM5 (http://www.fil.ion.ucl.ac.uk/spm). First, each participant's functional dataset was slice-time corrected, motion corrected, and unwarped, and then coregistered to the anatomical data. After segmentation, the data were spatially normalized into the standard MNI atlas space. Data were then smoothed with an $8 \mathrm{~mm}$ FWHM Gaussian kernel and high-pass filtered during statistical analysis. We applied the general linear model for serially autocorrelated data (Friston et al., 1995) and included one covariate for each task type in relation to the preceding trial: task repetition/hand repetition, task repetition/hand switch, task switch/hand repetition, and task switch/hand switch. Note that the present report focuses on task switching, while differences between task switching and hand switching will be reported separately. In addition, we included one covariate for error trials. To protect the whole-brain analysis against falsepositive activations, we used a double-threshold approach, that is, combining a voxel-based threshold with a minimum cluster size (Forman et al., 1995). This nonarbitrary voxel cluster size was determined on the basis of a Monte Carlo simulation (10,000 iterations) determined with AFNI's AlphaSim tool (Ward, 2000). We determined the minimal cluster size for an individual voxel height threshold of $T>2.67(p<$ 0.005 , uncorrected) to ensure an overall imagewise false-positive rate of $5 \%$. Based on our interest in DRD2 modulations of frontostriatal circuits, we simulated the cluster threshold within a masked volume including the frontal lobes and the dorsal part of the striatum (caudate nucleus, putamen). Anatomical masks were created using the frontal hemisphere mask and the automated anatomical labeling mask for the dorsal striatum (Tzourio-Mazoyer et al., 2002) in the WFU Pickatlas toolbox (Maldjian et al., 2003), restricting the included voxels to the group brain mask generated by SPM during statistical analysis. This resulted in a cluster threshold of 103 voxels. Activations exceeding this double threshold are therefore considered to be activated at an experimentwise threshold of $p<0.05$, corrected for multiple comparisons. Analyses of the interaction between switching condition and DRD2/ANKK1-TaqIa allele group were restricted to task switching-related brain regions by applying an inclusive mask from the whole-group task switching contrast. In this masked analyses, allele group effects were identified using a cluster size threshold that was adjusted to the new search volume. An additional Monte Carlo simulation for the masked volume resulted in a cluster threshold of $k>7$ voxels for $p<0.005$, corresponding to $p<0.05$, corrected. The parameter estimates of all voxels showing a significant switching by allele group interaction were then analyzed offline to illustrate the directionality of the interaction effect. Given that this secondary ROI analysis is not used for inference but merely for resolving the nature of the interaction, this analysis is not subject to non-independence error (Poldrack and Mumford, 2009).

Functional connectivity analysis. To explore how frontostriatal systems are modulated by DRD2 density, we compared the functional connectivity between prefrontal cortex and striatum between the two genetic groups using the method of psychophysiological interactions (Friston et al., 1997). The aim of a psychophysiological interaction (PPI) analysis is to explain neural responses in one brain region in terms of the interaction between the neural responses in another brain region and a specific psychological context. We used a prefrontal cluster of activation that in-
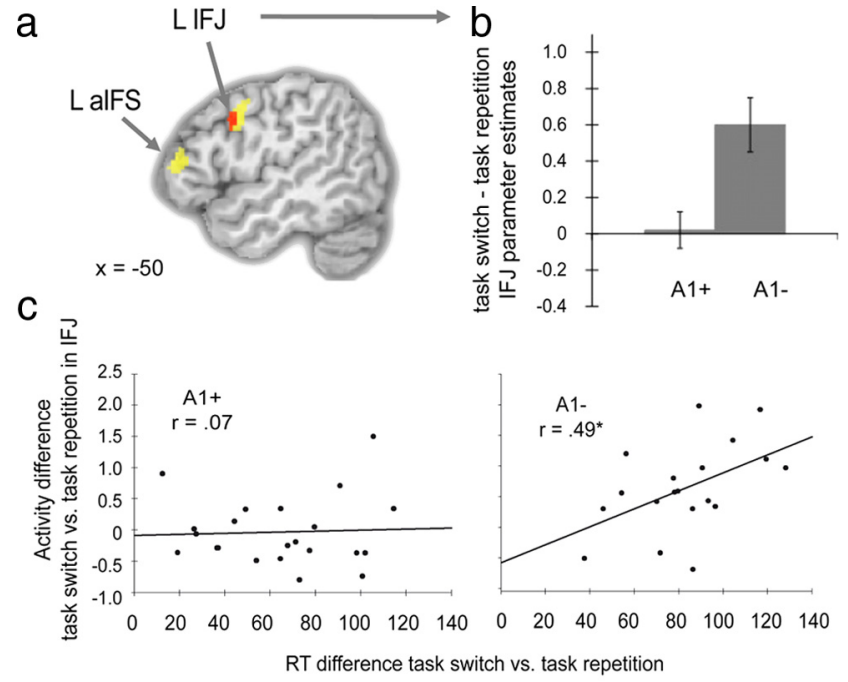

d

Functional connectivity (IFJ seed) A1- > A1+ for Task Switch > Repetition

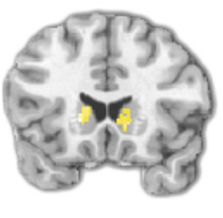

$y=+20$

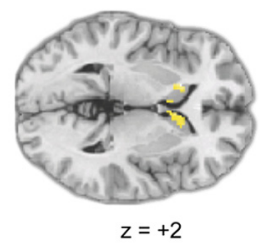

Figure 2. $\quad \boldsymbol{a}$, Across groups (yellow color code), task-switching-related activity was present in lateral prefrontal regions (thresholded at $p<0.005, k>103$ voxels, in frontostriatal anatomical mask). Within the IFJ, a subregion showed an interaction effect with DRD2/ANKK1-Taq1a (red color code). $\boldsymbol{b}$, The interaction reflects switching effects in the IFJ subcluster for A1 - individuals that are not present in A1 + individuals. Error bars, SEM. c, IFJ activity was correlated with behavioral switching costs in $A 1$ - but not $A 1+$ individuals $\left({ }^{*} p<\right.$ 0.05). $\boldsymbol{d}$, Increased task-switching-related functional connectivity of the IFJ with the dorsal striatum for $\mathrm{A} 1$ - compared to $\mathrm{A} 1+$ individuals (in dorsal striatum mask, $p<0.005, k>74$ voxel). alFS, Anterior inferior frontal sulcus.

cluded voxels showing the task switch versus repetition effect as seed region, and calculated the psychophysiological interaction term as the product of the mean time course in this region and the respective psychological variable (i.e., task switch vs repetition). All three variables (time course in seed region, psychological variable, and interaction term) were entered into a new general linear model for each subject. Then, two-sample $t$ tests were performed to compare the parameter estimates of the interaction term between the DRD2 groups, which effectively results in a three-way interaction of seed time course, task switching effect, and DRD2 group. We restricted the analysis to the striatum by applying an anatomical mask of this region (Tzourio-Mazoyer et al., 2002) using the WFU Pickatlas (Maldjian et al., 2003). As above, based on a Monte Carlo simulation for the striatal search volume, we combined a voxelbased threshold of $p<0.005$ with a minimum cluster size of 74 voxels to yield a corrected $p<0.05$.

\section{Results}

\section{Task switching}

As expected, task switching elicited performance costs, i.e., increased response times $\left(74 \pm 4.3 \mathrm{~ms}\right.$, SEM; $t_{(40)}=16.18, p<$ 0.001 ) (Fig. $1 b)$ and error rates $\left(2.5 \%, \pm 0.6 ; t_{(40)}=4.07, p<\right.$ 0.001 ) (Fig. $1 b$ ) compared to task repetitions. In addition, activity in lateral prefrontal brain regions associated with cognitive control was increased for switch relative to repetition trials (Fig. $2 a$, Table 1). The two clusters within the frontostriatal search volume exceeding the statistical threshold were located in the left inferior frontal junction (IFJ) of the precentral and inferior frontal sulci 
Table 1. Anatomical location and MNI coordinates for task switch versus repetition contrast across DRD2/ANKK1-Taqla groups in frontostriatal search volume $(p<0.005, k>103)$

\begin{tabular}{lllllllll}
\hline & & \multicolumn{9}{l}{ MNI coordinates } & & \\
\cline { 5 - 6 } Region & Hem & BA & $x$ & $y$ & $z$ & & $t$ value & Cluster size \\
\hline Inferior frontal junction & $\mathrm{L}$ & $6 / 8 / 44$ & -54 & 8 & 38 & 4.31 & 119 \\
Inferior frontal sulcus & $\mathrm{L}$ & 46 & -50 & 42 & 14 & 4.04 & 166 \\
\hline
\end{tabular}

Hem, Hemisphere (L, left; $R$, right); BA, Brodmann's area.

and in the anterior portion of the left inferior frontal sulcus. No evidence for striatal involvement in task switching across groups was present.

\section{DRD2/ANKK1-TaqIa association with task switching effects}

Importantly, behavioral switching effects were modulated by DRD2/ANKK1-TaqIa. Error rates were generally increased in $\mathrm{A} 1-(13.7 \pm 1.2)$ compared to A1 + individuals $(10.7 \pm 1.1)$, albeit not significantly $\left(t_{(39)}=1.81, p=0.08\right)$, but this DRD2/ ANKK1-TaqIa effect did not interact with the task switch versus repetition effect. The interaction between switching condition (repeat vs switch) and DRD2/ANKK1-TaqIa allele-group (i.e., $\mathrm{A} 1+\mathrm{vs} \mathrm{A} 1-$ ) on response times $\left(t_{(39)}=2.18, p=0.03\right.$ ) (Fig. $1 b$, see also supplemental Table S1, available at www.jneurosci.org as supplemental material) shows increased switching costs for A1participants $(83 \pm 5.6 \mathrm{~ms})$ compared to $\mathrm{A} 1+(65 \pm 6.4 \mathrm{~ms})$. Cohen's $d$ of 0.68 indicates that this is a medium to large effect (Cohen, 1992).

In accordance with these allelic effects on performance, prefrontal task-switching-related brain activations were differentially modulated depending on DRD2 density. The interaction analysis between switching condition and DRD2/ANKK1-TaqIa allele-group (inclusively masked by the whole-group switching contrast; see Materials and Methods) indicates that within the task-related prefrontal network described above, exclusively a subregion of the IFJ cluster $(x=-50, y=+12, z=+36 ; 28$ voxels; $\left.T_{\max }=3.46\right)$ showed stronger switching-related activity for $\mathrm{A} 1$ - than for $\mathrm{A} 1+$ individuals (red color code in Fig. 2a). Resolving this interaction effect (Fig. $2 b$, see also supplemental Table S2, available at www.jneurosci.org as supplemental material) indicates increased activity during task switches compared to repetitions in this subcluster only in the A1 - group but not in $\mathrm{A} 1+$ individuals. No region showed increased switching-related activity for $\mathrm{A} 1+$ compared to $\mathrm{A} 1$ - individuals.

As D2 receptors are most abundant in the striatum, which, however, did not show any robust switching-related activity in our sample of 48 participants, we additionally applied an ROI approach using an anatomical mask of the dorsal striatum (caudate nucleus and putamen) (Tzourio-Mazoyer et al., 2002). Interaction effects of DRD2/ANKK1-TaqIa and switching condition in these two regions could be detected only when lowering the statistical threshold as low as $p<0.05$, uncorrected, with one cluster in the right caudate nucleus $(x=+14, y=+12$, $z=+4 ; 79$ voxels; $\left.T_{\max }=2.38\right)$, one in the left putamen $(x=$ $-18, y=0, z=+10 ; 36$ voxels; $\left.T_{\max }=2.56\right)$, and one in the right putamen $\left(x=+28, y=+18, z=+6\right.$; 35 voxels; $\left.T_{\max }=2.33\right)$. While we do not consider these to be robust effects, it is noteworthy that the striatal ROIs show the same general pattern of increased switching costs in A1 - individuals as revealed by separate ROI analyses for the voxels of the caudate and the putamen, respectively. This analysis additionally revealed a main effect of DRD2 group in the caudate cluster $\left(F_{(1,46)}=9.53, p=0.003\right)$, indicating generally increased caudate activity in individuals with presumably low DRD2 density $(\mathrm{A} 1+)$.

The DRD2/ANKK1-TaqIa modulation of task-switchingrelated IFJ activation was further supported by differential correlations of the IFJ switching effect and switching costs in response times. As shown in Figure $2 c$, a correlation between the IFJ activation and response time switch costs in the whole group $(r=$ $0.38, p=0.02)$ was completely driven by the A $1-$ group $(r=$ $0.49, p=0.03$, right panel), while the A1+ group showed no such correlation $(r=0.07, p=0.77$, left panel). To test whether brainbehavior correlations for $\mathrm{A} 1+$ individuals were present in any other region, we further analyzed task-switching effects in the $\mathrm{A} 1+$ group, including the behavioral switching costs as a covariate for a whole-brain analysis. This analysis indicated that in the $\mathrm{A} 1+$ group switching-related activity $(p<0.005$, uncorrected $)$ in a circumscribed cluster in the left anterior IFS region $(x=$ $-52, y=+44, z=+8 ; 17$ voxels; $\left.T_{\max }=3.95\right)$ correlated with behavioral switching costs. This cluster was located slightly anterior to the whole-group cluster in the anterior IFS. While this correlation effect clearly failed to reach our significance criterion, this effect provides initial evidence of how A1+ participants coped with the task switching demand.

\section{Prefrontal-striatal coupling}

Given that D2 receptors are most abundant in the striatum (Pohjalainen et al., 1998) and we therefore reasoned that the prefrontal allele group effects should be associated with striatal signaling (Strafella et al., 2001; Bilder et al., 2004), we used PPI analysis (Friston et al., 1997) to examine how prefrontal DRD2/ ANKK1-TaqIa modulations of switching-related IFJ activation interacted with activity changes in the striatum. To this end, PPI results were assessed in an anatomically restricted region of the dorsal striatum (caudate nucleus and putamen; see Materials and Methods). In line with the hypothesized dorsal frontostriatal circuit for rule-based (as opposed to reward-dependent) flexibility, we observed that switching-specific functional connectivity between the IFJ and the caudate nucleus (i.e., increased frontostriatal coupling for switching relative to task repetition) was significantly associated with DRD2/ANKK1-TaqIa. Left $\left(x=-18, y=22, z=0 ; 132\right.$ voxels; $\left.T_{\max }=4.18\right)$ and right $\left(x=6, y=10, z=-8 ; 218\right.$ voxels; $\left.T_{\max }=4.09\right)$ caudate were functionally more strongly coupled with the IFJ for task switches compared to task repetitions in A1- individuals compared to $\mathrm{A} 1+$ (Fig. 2d). Further regions outside this search volume that also showed increased functional coupling for A1 - individuals are listed in supplemental Table S3, available at www.jneurosci.org as supplemental material).

\section{Haplotype analysis}

To further substantiate the present findings, we reanalyzed the data considering not only the DRD2/ANKK1-TaqIa polymorphism, but also two additional polymorphisms related to the D2 receptor (Fig. 3). While the DRD2 c957t (rs6277) single nucleotide polymorphism (SNP) is also associated with changes in D2 receptor availability (Pohjalainen et al., 1998), the DRD2 rs2283265 SNP affects the ratio of presynaptic versus postsynaptic DRD2 subtypes (Zhang et al., 2007). In the present sample, five different haplotypes could be identified (see Table 2). Based on a recent study from our laboratory (Markett et al., 2010), we regrouped the participants according to the presence versus absence of the TCT haplotype block [rs1800497/Taq-I a: T+ $(\mathrm{A} 1+)$; rs6277/c957t: C+; rs2283265; T+ ]. Carriers of the TCT haplotype can be assumed to show lower D2 receptor densities 

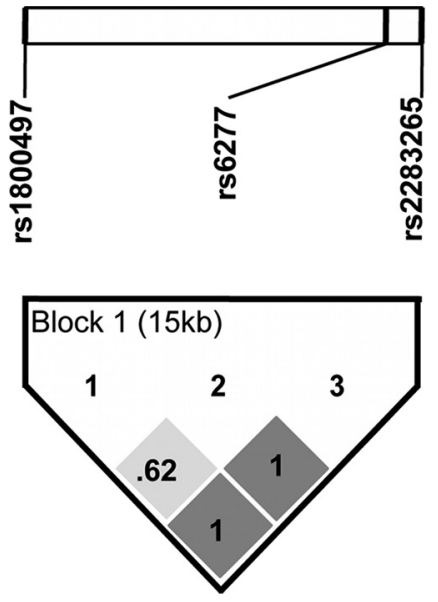

Figure 3. The haplotype block spanning the DRD2/ANKK1-Taqla SNP (rs1800497) and two other SNPs on the DRD2 gene (i.e., DRD2 c957t (rs6277) and rs2283265). Values in the diamonds represent $D^{\prime}$ values, a measure for the linkage disequilibrium between SNPs (see also Table 2 for frequencies).

\section{Table 2. Observed and expected haplotype frequencies as reconstructed with} PHASE

\begin{tabular}{llcc}
\hline & Haplotype & Observed & Expected \\
\hline 1 & CCG & 24 & 25 \\
2 & CCT & 0 & 0 \\
3 & CTG & 46 & 45 \\
4 & CTT & 0 & 0 \\
5 & TCG & 4 & 3 \\
6 & TCT & 18 & 18 \\
7 & TTG & 4 & 5 \\
8 & TTT & 0 & 0 \\
\hline
\end{tabular}

In the haplotype column, the triplets refer to the genetic variant of the (1) DRD2/ANKK1-Taqla (rs1800497; T:A1+, C: A1-); (2) DRD2 c957t (rs6277); and (3) DRD2 rs22832565.

and a shifted proportion of presynaptic to postsynaptic D2 receptors. Accordingly, this analysis establishes whether the obtained effects reported above are specific to the DRD2/ANKK1-TaqIa polymorphism or whether they are more generally related to genetically determined individual differences in DRD2 availability. The analysis revealed that 17 individuals were carriers of the TCT haplotype block (one of whom homozygous). When contrasting these individuals with the remaining 31 noncarriers of the TCT haplotype block, we obtained results very similar to those from the comparison of A1 + versus A1 - individuals: behaviorally, TCT carriers showed reduced task-switching RT costs $(62.8 \pm 6.7)$ compared to TCT noncarriers $\left(79.1 \pm 5.7 ; t_{(39)}=\right.$ $1.79, p=0.08)$.

The analysis of haplotype $\times$ switching effects within the inclusive task switching mask also revealed a subcluster of the IFJ that was differentially modulated by haplotype (Fig. 4a) $(x=$ $-50, y=12, z=36 ; 48$ voxels; $T_{\max }=4.88$ ). Note that this subcluster was nearly twice the size of the pure DRD2/ANKK1TaqIa interaction cluster and yielded an even higher maximal $t$ value. Illustrating the directionality of the interaction effect in the voxels of this subcluster indicated higher task-switching effects in the IFJ for TCT noncarriers than for TCT carriers (Fig. $4 a$ and supplemental Table S4, available at www.jneurosci.org as supplemental material). The correlation of IFJ activity with behavioral switching effects was also greater for TCT noncarriers $(r=0.41$, $p=0.04)$ than for TCT carriers $(r=-0.25, p=0.38)$. Finally, the results of the PPI analysis indicated that, albeit with a reduced cluster size, the TCT noncarriers also showed increased switching-specific functional coupling between the IFJ and the left $\left(x=-16, y=24, z=2 ; 29\right.$ voxels; $\left.T_{\max }=3.79\right)$ and right $\left(x=20, y=24, z=-2 ; 39\right.$ voxels; $\left.T_{\max }=3.53\right)$ caudate when compared to TCT carriers (Fig. $4 b$ ).

\section{Discussion}

The present results indicate that individual differences in the implementation of rule-based switching processes strongly depend on dopamine D2-receptor-mediated neural processing. Noncarriers of the DRD2/ANKK1-TaqIa A1 allele (A1-) with presumably higher D2 receptor densities, show greater switching costs behaviorally, being directly related to increased task-switching activity in the lateral prefrontal cortex. Increased processing resources invested by these individuals are further reflected in increased prefrontal coupling with the dorsal striatum during switching. A haplotype analysis involving three genetic polymorphisms modulating DRD2 availability (i.e., rs1800497, rs6277, and rs2283265) confirmed these results, providing strong support for our hypothesis that rule-based cognitive flexibility without rewards depends on DRD2 availability. These results indicate that in healthy humans, high DRD2 density may indeed be disadvantageous for certain cognitive demands by increasing the invested effort for successful performance.

Several clinical studies point to an association of changes in the DRD2 system and cognitive inflexibility. In particular, high DRD2 density is a major component in the pathophysiology of schizophrenia (Wong et al., 1986; Abi-Dargham et al., 2000). While many studies show impaired cognitive flexibility in schizophrenic patients (Rodríguez-Sánchez et al., 2005; Thoma et al., 2007), only recently the link between high DRD2 density and cognitive inflexibility was established: a genetic mutation study showed that the overexpression of striatal D2 receptors in transgenic mice was directly related to reversal learning deficits (Kellendonk et al., 2006).

In addition, the A1 allele of the DRD2/ANKK1-TaqIa polymorphism has been associated with other disorders characterized by cognitive and behavioral impulsivity such as addiction (Bowirrat and Oscar-Berman, 2005), compulsive gambling (Comings et al., 1996), or attention deficit hyperactivity disorder (Comings et al., 1991; Kirley et al., 2002). The lowered threshold for switching to addiction- or distracter-related behaviors in these disorders fits nicely to the greater task-switching efficiency in our Al carriers, who seem to adopt novel behavioral options more readily. The crucial difference lies in the healthy A1 carriers' ability for goal-directed flexibility-while impulsivity and inattention as experienced by these patients, in contrast, is mostly dysfunctional. Note, however, that the processing advantage of A1 carriers seems to vanish when behavior is reinforced by feedback or reward. During feedback-based learning, Al carriers are less proficient in adjusting their behavior based on feedback in previous trials (Klein et al., 2007; Jocham et al., 2009). Thus, feedback might actually alter the mechanisms involved in cognitive flexibility substantially, most likely due to processes involved in anticipating outcomes. This fundamental difference is most directly reflected in the involvement of ventral as compared to dorsal frontostriatal neural systems (Alexander et al., 1986).

The exact neural mechanisms of how individual differences in DRD2 density result in differences in prefrontal activity are not well understood yet. Critically, the D2 receptor is most abundant in the striatum (Pohjalainen et al., 1998). Several loops between the striatum and prefrontal cortex have been described (Alexander et al., 1986), and computational models of frontostriatal interactions (Cohen et al., 1996; Frank et al., 2001) make it 
plausible that the striatum is involved in signaling whether information in circumscribed prefrontal regions should be updated to reconfigure the relevant task set according to the task cue (Meiran, 1996). This gating signal is assumed to depend on the striatal dopamine level (Cohen et al., 1996; Braver and Cohen, 1999), with stronger signals associated with more dopamine in the striatum leading to faster and less effortful updating in the prefrontal cortex (Durstewitz and Seamans, 2008). While this seems inconsistent with the finding of greater switching costs for participants with greater receptor densities, our data can be reconciled with this model based on recent evidence from a PET ligand study. Laakso et al. (2005) showed that striatal dopamine synthesis is actually increased in $\mathrm{A} 1+$ individuals, i.e., in persons with reduced DRD2 density, presumably due to altered autoreceptor functions. This receives support from our observation of generally increased striatal BOLD signals in A1+ compared to A1- individuals. In addition, this is consistent with recent evidence on the role of genetic differences in prefrontal dopamine availability for cognitive flexibility (Krugel et al., 2009; Colzato et al., 2010). In these studies, individuals with low prefrontal dopamine levels were behaviorally more flexible and, importantly, this was associated with increased striatal signaling, presumably associated with stronger dopaminergic burst firing related to reward (Bilder et al., 2004; Krugel et al., 2009). These findings together with the present results thus support previous assumptions on an inverse relationship between prefrontal and striatal dopamine activity (Kolachana et al., 1995).

The study of Laakso et al. (2005) provides an important link between the finding of impaired switching performance and increased neural effort for high DRD2 density in the present study and impaired task switching for individuals under the DRD2 antagonist sulpiride (Mehta et al., 2004). The putative adaptive increase in dopamine synthesis in Al carriers might explain why genetically determined low DRD2 density is not necessarily associated with decreased DRD2 signaling as evoked by transient pharmacological interventions. Instead, adaptively increased dopamine signaling in A1 carriers might evoke a more salient gating signal, facilitating the updating of task representations in prefrontal cortex.

Alternatively, less strong IFJ signaling might be needed in A1 carriers because the relevant task representations are generally in a different activation state, e.g., because multiple representations are kept in a more accessible state throughout the experiment. This could explain the missing association between IFJ activity and behavioral switching costs in Al carriers that was present in A1 - individuals. The exploratory analysis of brain-behavior correlations in the $\mathrm{A} 1+$ group revealed a small anterior inferior frontal cluster showing an association with behavioral switching costs. As the size of this cluster was below the threshold for a whole-brain analysis, we only tentatively consider this post hoc finding as evidence that A1 carriers, based on their genetic constitution, might use a fundamentally different strategy for switching between tasks. Previous findings showed anterior prefrontal involvement for endogenously triggered task switching (Forstmann et al., 2005) and for mixing two tasks in a task-switching setting compared to single-task performance (Braver et al., 2003). In addition, the simultaneous processing of multiple goals involves anterior prefrontal cortex (Koechlin et al., 2003). Accordingly, instead of involving the IFJ for sequentially uploading the relevant task set based on task cues, A1 carriers might use a more abstract strategy, e.g., keeping both task representations in an activated state and focusing temporally more on one or the other resulting in better task performance and overall greater neural efficiency. Further studies manipulating potential strategies experimentally might further elucidate these potential interpretations.

The greater efficiency of A1 carriers is further supported by the relatively weaker functional connectivity between the dorsal striatum and the IFJ region during switching. Various studies showed increased functional connectivity in clinical populations associated with performance deficits in cognitive tasks (Schlösser et al., 2003, 2008; Meyer-Lindenberg et al., 2005; Shaw et al., 2009). In addition, decreased task-related coupling in clinical conditions such as schizophrenia is presumably compensated by increased connectivity in other networks (Friston, 1998; Stephan et al., 2006; Spoletini et al., 2009). Model-free analyses of connectivity patterns, e.g., using independent component analysis, could provide a more complete picture of differences in connectivity patterns between genetic groups to evaluate this compensation hypothesis directly.

While switching-specific functional connectivity between striatum and IFJ differed between $\mathrm{A} 1+$ and A1 - individuals, we found only subthreshold switching-related activity differences in the striatum. This is different from previous findings on the role of DRD2 in switching between visual stimulus categories (Cools et al., 2007) and reward- or feedback-based learning (Klein et al., 2007; Jocham et al., 2009), and might reflect the prefrontal dominance in intentional nonrewarded switching processes. Cools et al. (2007) administered the D2 receptor agonist bromocriptine in healthy individuals with different degrees of impulsivity who had to switch between stimulus categories rather than, as in the present study, between task rules based on a cue. Trait impulsivity has been associated with low D2 receptor binding in the striatum, most likely reflecting low striatal D2 receptor density (Dalley et al., 2007). Striatal activity and behavioral switching performance were modulated by bromocriptine in high impulsive individuals only, showing that for successful stimulus-based switching, optimal DRD2 activity in the striatum is crucial. Here, we show that for flexibly applying different abstract rules to the same stimuli, DRD2 effects are mainly present in a region of the lateral prefrontal cortex previously associated with the updating of task representations (Brass et al., 2005; Derrfuss et al., 2005). The selective coupling between PFC and dorsal striatum, however, indicates 
that the PFC is not working in isolation and thus supports the outlined striatofrontal gating hypothesis.

In sum, the present results provide strong evidence that differences in the availability of D2 receptors contribute to individual differences in intentional cognitive flexibility associated with dorsal frontostriatal circuits. These findings form the basis for a more general framework on the role of D2 activity in frontostriatal circuits and extend our current knowledge concerning the role of D2 receptor-related activity for cognitive flexibility, which was previously mainly based on reward-based flexibility.

\section{References}

Abi-Dargham A, Rodenhiser J, Printz D, Zea-Ponce Y, Gil R, Kegeles LS, Weiss R, Cooper TB, Mann JJ, Van Heertum RL, Gorman JM, Laruelle M (2000) Increased baseline occupancy of D2 receptors by dopamine in schizophrenia. Proc Natl Acad Sci U S A 97:8104-8109.

Alexander GE, DeLong MR, Strick PL (1986) Parallel organization of functionally segregated circuits linking basal ganglia and cortex. Annu Rev Neurosci 9:357-381.

Bilder RM, Volavka J, Lachman HM, Grace AA (2004) The catechol-0methyltransferase polymorphism: relations to the tonic-phasic dopamine hypothesis and neuropsychiatric phenotypes. Neuropsychopharmacology 29:1943-1961.

Bowirrat A, Oscar-Berman M (2005) Relationship between dopaminergic neurotransmission, alcoholism, and reward deficiency syndrome. Am J Med Genet B Neuropsychiatr Genet 132B:29-37.

Brass M, von Cramon DY (2002) The role of the frontal cortex in task preparation. Cereb Cortex 12:908-914.

Brass M, Derrfuss J, Forstmann B, von Cramon DY (2005) The role of the inferior frontal junction area in cognitive control. Trends Cogn Sci 9:314-316.

Braver TS, Cohen JD (1999) Dopamine, cognitive control, and schizophrenia: the gating model. Prog Brain Res 121:327-349.

Braver TS, Reynolds JR, Donaldson DI (2003) Neural mechanisms of transient and sustained cognitive control during task switching. Neuron 39:713-726.

Cohen J (1992) A power primer. Psychol Bull 112:155-159.

Cohen JD, Braver TS, O’Reilly RC (1996) A computational approach to prefrontal cortex, cognitive control and schizophrenia: recent developments and current challenges. Philos Trans R Soc Lond B Biol Sci 351:1515-1527.

Colzato LS, Waszak F, Nieuwenhuis S, Posthuma D, Hommel B (2010) The flexible mind is associated with the catechol-O-methyltransferase (COMT) Val158Met polymorphism: evidence for a role of dopamine in the control of task-switching. Neuropsychologia 48:2764-2768.

Comings DE, Comings BG, Muhleman D, Dietz G, Shahbahrami B, Tast D, Knell E, Kocsis P, Baumgarten R, Kovacs BW, Levy DL, Smith M, Borison RL, Durrell Evans D, Klein DN, MacMurray J, Tosk J, Sverd J, Gysin R, Flanagan S (1991) The dopamine D2 receptor locus as a modifying gene in neuropsychiatric disorders. JAMA 266:1793-1800.

Comings DE, Rosenthal RJ, Lesieur HR, Rugle LJ, Muhleman D, Chiu C, Dietz G, Gade R (1996) A study of the dopamine D2 receptor gene in pathological gambling. Pharmacogenetics 6:223-234.

Cools R, Robbins TW (2004) Chemistry of the adaptive mind. Philos Transact A Math Phys Eng Sci 362:2871-2888.

Cools R, Barker RA, Sahakian BJ, Robbins TW (2001) Mechanisms of cognitive set flexibility in Parkinson's disease. Brain 124:2503-2512.

Cools R, Sheridan M, Jacobs E, D’Esposito M (2007) Impulsive personality predicts dopamine-dependent changes in frontostriatal activity during component processes of working memory. J Neurosci 27:5506-5514.

Dalley JW, Fryer TD, Brichard L, Robinson ES, Theobald DE, Lääne K, Peña Y, Murphy ER, Shah Y, Probst K, Abakumova I, Aigbirhio FI, Richards HK, Hong Y, Baron JC, Everitt BJ, Robbins TW (2007) Nucleus accumbens $\mathrm{D} 2 / 3$ receptors predict trait impulsivity and cocaine reinforcement. Science 315:1267-1270.

Derrfuss J, Brass M, Neumann J, von Cramon DY (2005) Involvement of the inferior frontal junction in cognitive control: meta-analyses of switching and Stroop studies. Hum Brain Mapp 25:22-34.

Durstewitz D, Seamans JK (2008) The dual-state theory of prefrontal cortex dopamine function with relevance to catechol-o-methyltransferase genotypes and schizophrenia. Biol Psychiatry 64:739-749.
Floresco SB, Magyar O, Ghods-Sharifi S, Vexelman C, Tse MT (2006) Multiple dopamine receptor subtypes in the medial prefrontal cortex of the rat regulate set-shifting. Neuropsychopharmacology 31:297-309.

Forman SD, Cohen JD, Fitzgerald M, Eddy WF, Mintun MA, Noll DC (1995) Improved assessment of significant activation in functional magnetic resonance imaging (fMRI): use of a cluster-size threshold. Magn Reson Med 33:636-647.

Forstmann BU, Brass M, Koch I, von Cramon DY (2005) Internally generated and directly cued task sets: an investigation with fMRI. Neuropsychologia 43:943-952.

Frank MJ, Loughry B, O’Reilly RC (2001) Interactions between frontal cortex and basal ganglia in working memory: a computational model. Cogn Affect Behav Neurosci 1:137-160.

Friedman NP, Miyake A, Young SE, Defries JC, Corley RP, Hewitt JK (2008) Individual differences in executive functions are almost entirely genetic in origin. J Exp Psychol Gen 137:201-225.

Friston KJ (1998) The disconnection hypothesis. Schizophr Res 30:115-125.

Friston KJ, Holmes AP, Worsley KJ, Poline JP, Frith CD, Frackowiak RS (1995) Statistical parametric maps in functional imaging: a general linear approach. Hum Brain Mapp 2:189-210.

Friston KJ, Buechel C, Fink GR, Morris J, Rolls E, Dolan RJ (1997) Psychophysiological and modulatory interactions in neuroimaging. Neuroimage 6:218-229.

Fuster JM (2001) The prefrontal cortex-an update: time is of the essence. Neuron 30:319-333.

Goldberg TE, Weinberger DR (1988) Probing prefrontal function in schizophrenia with neuropsychological paradigms. Schizophr Bull 14:179-183.

Gu BM, Park JY, Kang DH, Lee SJ, Yoo SY, Jo HJ, Choi CH, Lee JM, Kwon JS (2008) Neural correlates of cognitive inflexibility during task-switching in obsessive-compulsive disorder. Brain 131:155-164.

Jersild A (1927) Mental set and shift. Arch Psychol 14:81.

Jocham G, Klein TA, Neumann J, von Cramon DY, Reuter M, Ullsperger M (2009) Dopamine DRD2 polymorphism alters reversal learning and associated neural activity. J Neurosci 29:3695-3704.

Kehagia AA, Cools R, Barker RA, Robbins TW (2009) Switching between abstract rules reflects disease severity but not dopaminergic status in Parkinson's disease. Neuropsychologia 47:1117-1127.

Kellendonk C, Simpson EH, Polan HJ, Malleret G, Vronskaya S, Winiger V, Moore H, Kandel ER (2006) Transient and selective overexpression of dopamine $\mathrm{D} 2$ receptors in the striatum causes persistent abnormalities in prefrontal cortex functioning. Neuron 49:603-615.

Kirley A, Hawi Z, Daly G, McCarron M, Mullins C, Millar N, Waldman I, Fitzgerald M, Gill M (2002) Dopaminergic system genes in ADHD: toward a biological hypothesis. Neuropsychopharmacology 27:607-619.

Klein TA, Neumann J, Reuter M, Hennig J, von Cramon DY, Ullsperger M (2007) Genetically determined differences in learning from errors. Science 318:1642-1645.

Koechlin E, Ody C, Kouneiher F (2003) The architecture of cognitive control in the human prefrontal cortex. Science 302:1181-1185.

Kolachana BS, Saunders RC, Weinberger DR (1995) Augmentation of prefrontal cortical monoaminergic activity inhibits dopamine release in the caudate nucleus: an in vivo neurochemical assessment in the rhesus monkey. Neuroscience 69:859-868.

Krugel LK, Biele G, Mohr PN, Li SC, Heekeren HR (2009) Genetic variation in dopaminergic neuromodulation influences the ability to rapidly and flexibly adapt decisions. Proc Natl Acad Sci U S A 106:17951-17956.

Laakso A, Pohjalainen T, Bergman J, Kajander J, Haaparanta M, Solin O, Syvalahti E, Hietala J (2005) The A1 allele of the human D2 dopamine receptor gene is associated with increased activity of striatal L-amino acid decarboxylase in healthy subjects. Pharmacogenet Genomics 15:387-391.

Maldjian JA, Laurienti PJ, Kraft RA, Burdette JH (2003) An automated method for neuroanatomic and cytoarchitectonic atlas-based interrogation of fMRI data sets. Neuroimage 19:1233-1239.

Markett SA, Montag C, Reuter M (2010) The association between dopamine DRD2 polymorphisms and working memory capacity is modulated by a functional polymorphism on the nicotinic receptor gene CHRNA4. J Cogn Neurosci 22:1944-1954.

Mehta MA, Manes FF, Magnolfi G, Sahakian BJ, Robbins TW (2004) Impaired set-shifting and dissociable effects on tests of spatial working memory following the dopamine D2 receptor antagonist sulpiride in human volunteers. Psychopharmacology (Berl) 176:331-342. 
Meiran N (1996) Reconfiguration of processing mode prior to task performance. J Exp Psychol Learn Mem Cogn 22:1423-1442.

Meyer-Lindenberg A, Weinberger DR (2006) Intermediate phenotypes and genetic mechanisms of psychiatric disorders. Nat Rev Neurosci 7:818-827.

Meyer-Lindenberg AS, Olsen RK, Kohn PD, Brown T, Egan MF, Weinberger DR, Berman KF (2005) Regionally specific disturbance of dorsolateral prefrontal-hippocampal functional connectivity in schizophrenia. Arch Gen Psychiatry 62:379-386.

Miller EK, Cohen JD (2001) An integrative theory of prefrontal cortex function. Annu Rev Neurosci 24:167-202.

Miyake A, Friedman NP, Emerson MJ, Witzki AH, Howerter A, Wager TD (2000) The unity and diversity of executive functions and their contributions to complex "frontal lobe" tasks: a latent variable analysis. Cogn Psychol 41:49-100.

Monsell S (2003) Task switching. Trends Cogn Sci 7:134-140.

Noble EP (2000a) The DRD2 gene in psychiatric and neurological disorders and its phenotypes. Pharmacogenomics 1:309-333.

Noble EP (2000b) Addiction and its reward process through polymorphisms of the D2 dopamine receptor gene: a review. Eur Psychiatry 15:79-89.

Noble EP (2003) D2 dopamine receptor gene in psychiatric and neurologic disorders and its phenotypes. Am J Med Genet B Neuropsychiatr Genet 116B:103-125.

Noble EP, Gottschalk LA, Fallon JH, Ritchie TL, Wu JC (1997) D2 dopamine receptor polymorphism and brain regional glucose metabolism. Am J Med Genet 74:162-166.

Pohjalainen T, Rinne JO, Någren K, Lehikoinen P, Anttila K, Syvälahti EK, Hietala J (1998) The A1 allele of the human D2 dopamine receptor gene predicts low D2 receptor availability in healthy volunteers. Mol Psychiatry 3:256-260

Poldrack RA, Mumford JA (2009) Independence in ROI analysis: where is the voodoo? Soc Cogn Affect Neurosci 4:208-213.

Ritchie T, Noble EP (2003) Association of seven polymorphisms of the D2 dopamine receptor gene with brain receptor-binding characteristics. Neurochem Res 28:73-82.

Rodríguez-Sánchez JM, Crespo-Facorro B, Perez-Iglesias R, González-Blanch C, Alvarez-Jimenez M, Llorca J, Vázquez-Barquero JL (2005) Prefrontal cognitive functions in stabilized first-episode patients with schizophrenia spectrum disorders: a dissociation between dorsolateral and orbitofrontal functioning. Schizophr Res 77:279-288.

Schlösser RG, Wagner G, Koch K, Dahnke R, Reichenbach JR, Sauer H (2008) Fronto-cingulate effective connectivity in major depression: a study with fMRI and dynamic causal modeling. Neuroimage 43:645-655.
Schlösser R, Gesierich T, Kaufmann B, Vucurevic G, Stoeter P (2003) Altered effective connectivity in drug free schizophrenic patients. Neuroreport 14:2233-2237.

Shaw ME, Moores KA, Clark RC, McFarlane AC, Strother SC, Bryant RA, Brown GC, Taylor JD (2009) Functional connectivity reveals inefficient working memory systems in post-traumatic stress disorder. Psychiatry Res 172:235-241.

Spoletini I, Cherubini A, Di Paola M, Banfi G, Rüsch N, Martinotti G, Bria P, Rubino IA, Siracusano A, Caltagirone C, Spalletta G (2009) Reduced fronto-temporal connectivity is associated with frontal gray matter density reduction and neuropsychological deficit in schizophrenia. Schizophr Res 108:57-68.

Stelzel C, Basten U, Montag C, Reuter M, Fiebach CJ (2009) Effects of dopamine-related gene-gene interactions on working memory component processes. Eur J Neurosci 29:1056-1063.

Stephan KE, Baldeweg T, Friston KJ (2006) Synaptic plasticity and dysconnection in schizophrenia. Biol Psychiatry 59:929-939.

Stephens M, Smith NJ, Donnelly P (2001) A new statistical method for haplotype reconstruction from population data. Am J Hum Genet 68:978-989.

Strafella AP, Paus T, Barrett J, Dagher A (2001) Repetitive transcranial magnetic stimulation of the human prefrontal cortex induces dopamine release in the caudate nucleus. J Neurosci 21:RC157.

Thoma P, Wiebel B, Daum I (2007) Response inhibition and cognitive flexibility in schizophrenia with and without comorbid substance use disorder. Schizophr Res 92:168-180.

Tzourio-Mazoyer N, Landeau B, Papathanassiou D, Crivello F, Etard O, Delcroix N, Mazoyer B, Joliot M (2002) Automated anatomical labeling of activations in SPM using a macroscopic anatomical parcellation of the MNI MRI single-subject brain. Neuroimage 15:273-289.

Wallis JD (2007) Orbitofrontal cortex and its contribution to decisionmaking. Annu Rev Neurosci 30:31-56.

Ward B (2000) Simultaneous inference for fMRI data. AFNI 3dDeconvolve Documentation. Milwaukee, WI: Medical College of Wisconsin.

Wong DF, Wagner HN Jr, Tune LE, Dannals RF, Pearlson GD, Links JM, Tamminga CA, Broussolle EP, Ravert HT, Wilson AA, Toung JK, Malat J, Williams JA, O’Tuama LA, Snyder SH, Kuhar MJ, Gjedde A (1986) Positron emission tomography reveals elevated $\mathrm{D} 2$ dopamine receptors in drug-naive schizophrenics. Science 234:1558-1563.

Zhang Y, Bertolino A, Fazio L, Blasi G, Rampino A, Romano R, Lee ML, Xiao T, Papp A, Wang D, Sadée W (2007) Polymorphisms in human dopamine D2 receptor gene affect gene expression, splicing, and neuronal activity during working memory. Proc Natl Acad Sci U S A 104:2055220557. 